\section{Avaliação dos óbitos neonatais em recém-nascidos de muito baixo peso em quatro maternidades no Município do Rio de Janeiro, Brasil}

\author{
Evaluation of neonatal mortality in very low \\ birth weight infants in four maternity hospitals \\ in the Municipality of Rio de Janeiro, Brazil
}

José Luiz Muniz Bandeira Duarte ${ }^{1}$ Gulnar Azevedo Silva Mendonça ${ }^{2}$

\title{
Introdução
}

1 Faculdade de Ciências Médicas, Universidade do Estado do Rio de Janeiro, Rio de Janeiro, Brasil.

2 Instituto de Medicina Social, Universidade do Estado do Rio de Janeiro, Rio de Janeiro, Brasil.

Correspondência J. L. M. B. Duarte Faculdade de Ciências Médicas, Universidade do Estado do Rio de Janeiro. Rua Nelson Rodrigues 162, Rio de Janeiro, RJ 22793-260, Brasil. jlduarte@centroin.com.br bandeira@uerj.br

\begin{abstract}
The objective of this study was to describe the causes of neonatal mortality in four maternity hospitals in the Municipality of Rio de Janeiro, Brazil. The study included all very low birth weight newborns during the first complete 27 days of life. The distribution of live newborns and all very low birth weight newborns as well as the percentage of deaths per hospital and the totals were described. The study estimated total and specific neonatal mortality coefficients and assessed viability, time, and causes of death. The study sample consisted of 487 newborns. We found an $11.3 \%$ neonatal mortality rate (8.3\%o early neonatal and 2.9\%o late neonatal). Viability for all very low birth weight newborns was above 26 weeks' gestation and birth weight of 749g. Mortality was low in the first three days of life, mainly in the first day, and higher from the fourth to the 28th day. Sepsis was the main cause of neonatal mortality. Health systems managers should focus on: diagnosis and control of perinatal infections; decreasing neonatal hospital infection by reducing overcrowding in the nursery; and routine pathological investigation on all placentas in very low birth weight newborns as well as routine autopsies in case of death.
\end{abstract}

Infant Mortality; Very Low Birth Weight Infant; Cause of Death
A mortalidade infantil é um dos indicadores que melhor refletem as condições gerais de vida de uma população. Os estudos realizados no Brasil mostraram que a participação relativa do componente pós-neonatal na mortalidade infantil, durante os anos 90, apresentou uma redução considerável, o que torna proporcionalmente maior a participação do componente neonatal 1 . Nos anos 90, os estudos realizados no Rio Grande do Sul e no Rio de Janeiro 1,2 mostraram um maior predomínio de óbitos durante a primeira semana de vida, provocados por uma deficiente atenção à gestante, ao parto e ao recém-nascido.

A mortalidade neonatal no Município do Rio de Janeiro diminuiu de 15,2\%o, em 1994, para 10,5\%o, em 2002. Mas esse declínio ainda foi pequeno se o compararmos à tendência verificada nos países desenvolvidos. Esse fato aponta para a necessidade de realização de outros estudos que avaliem a qualidade do atendimento obstétrico e neonatal.

O componente neonatal precoce corresponde com cerca $52,3 \%$ dos óbitos ocorridos no primeiro ano de vida e quando se analisam apenas os recém-nascidos de muito baixo peso, aqueles que nasceram com peso inferior a $1.500 \mathrm{~g}$, esta contribuição se mostra mais elevada $(64,9 \%)^{3}$. Gomes 4, também no Município do Rio de Janeiro, encontrou resultados semelhantes. 
Nos Estados Unidos, Philip 5 estudou os dados entre os anos de 1981 e 1991, no intuito de averiguar se seria possível diminuir a mortalidade dos recém-nascidos com extremo baixo peso, aqueles com peso, ao nascimento, inferior a $1.000 \mathrm{~g}$. Considerando que viabilidade seria quando a sobrevivência sobrepujaria a mortalidade, os resultados demonstraram uma queda na mortalidade e uma diminuição da viabilidade, de 25-26 para 23-24 semanas de gestação. As reduções foram atribuídas ao uso de surfactante pulmonar e, portanto, a uma diminuição de morte por doença da membrana hialina. $\mathrm{O}$ autor refere que praticamente cessaram as mortes em recém-nascidos com peso superior a $1.000 \mathrm{~g}$.

O fator preditivo de sobrevivência em recém-nascidos com extremo baixo peso, independentemente do peso ao nascer, seria a sobrevida no quarto dia de vida 6 . Dos recémnascidos que estavam vivos no quarto dia de vida, cerca de $80,0 \%$ mostraram sobrevivência até a alta hospitalar. A mortalidade cumulativa mostrada foi de $47,0 \%$ no primeiro dia, $61,0 \%$ no segundo dia, $76,0 \%$ no terceiro dia e $81,0 \%$ no quarto dia. Após essa data a variação da sobrevida foi muito pequena. O estudo de Philip 5 mostrou, também em recém-nascidos com extremo baixo peso, o tempo de morte de $65,0 \%$ nas primeiras $24 \mathrm{~h}, 19,0 \%$ entre 1 e 7 dias, $8,0 \%$ entre 7 e 28 dias e 9,0\% após 28 dias.

Estudo realizado com os dados observados no período de 1981 a 1991, por Philip 5, mostrou que a causa mais freqüente de morte em recémnascidos com menos de $1.000 \mathrm{~g}$ foi a extrema prematuridade $(58,0 \%)$, seguida de problemas respiratórios $(24,4 \%)$, displasia broncopulmonar e malformações $(5,1 \%)$ e infecções $(4,0 \%)$.

Na Finlândia, Tommiska et al. 7, durante o período de 1996 a 1997, analisaram as principais causas de mortalidade em recém-nascidos com menos de 1.000 g e concluíram ser: doença da membrana hialina $(29,0 \%)$; imaturidade $(26,0 \%)$; hemorragia intracraniana graus III e IV (15,0\%); infecção (7,5\%); enterocolite necrosante $(6,0 \%)$; anomalias congênitas $(3,0 \%)$; e asfixia $(2,2 \%)$.

Para o Município do Rio de Janeiro, Gomes 4 , no seu estudo com dados coletados em quatro maternidades no período de quatro meses, durante o ano de 2000, mostrou o tempo de morte cumulativa no primeiro, segundo e sétimo dias de vida de $20,0 \%, 42,8 \%$ e $64,2 \%$, respectivamente. Encontrou como causas freqüentes de óbito a doença da membrana hialina $(51,4 \%)$, a infecção $(41,4 \%)$, a insuficiência respiratória e a falência de vários órgãos $(14,2 \%)$ e a asfixia $(12,8 \%)$.
A avaliação da qualidade do atendimento nos serviços de atenção terciária perinatal no âmbito do Município do Rio de Janeiro é fundamental para que se possa melhorar a assistência prestada.

Este estudo foi realizado para a obtenção de dados sobre a mortalidade em recém-nascidos de muito baixo peso utilizando como indicadores os coeficientes de mortalidade neonatal e seus componentes, a viabilidade, o tempo e as causas de óbitos. Espera-se com isso que as informações geradas possam contribuir para o aprimoramento de políticas assistenciais e preventivas voltadas aos períodos perinatal e neonatal.

\section{Material e métodos}

Foi realizado um estudo prospectivo longitudinal no qual foram incluídos todos os recémnascidos com peso, ao nascimento, igual/superior a $500 \mathrm{~g}$ e inferior a $1.500 \mathrm{~g}$ até completarem 27 dias de vida, em quatro maternidades localizadas no Município do Rio de Janeiro: (1) Hospital Universitário Pedro Ernesto da Universidade do Estado do Rio de Janeiro; (2) Instituto Fernandes Figueira do Ministério da Saúde; (3) Instituto Municipal da Mulher Fernando Magalhães; e (4) Hospital Maternidade Alexandre Fleming, sendo as duas últimas grandes maternidades municipais. Os dois primeiros serviços possuem residências credenciadas pelo Ministério da Educação para formação de médicos especializados em neonatologia. As duas últimas maternidades são aquelas com o segundo e terceiro maior número de atendimento no município. O estudo compreendeu o período entre 28 de agosto de 2001 a 28 de setembro de 2002.

Foram excluídos os recém-nascidos com idade gestacional inferior a 22 semanas ou portadores de malformações congênitas incompatíveis com a vida, como a anencefalia, a trissomia do 18, a agenesia renal, bem como as crianças transferidas para instituição não participante do estudo. Os recém-nascidos com malformações congênitas de elevado risco de vida, como a hérnia diafragmática, a cardiopatia congênita complexa, a atresia intestinal, a hidropsia e algum erro inato do metabolismo, foram incluídos no estudo.

O tamanho amostral foi calculado utilizando-se como parâmetros estatísticos um intervalo de confiança de $95 \%$ e poder de $80 \%$. Considerou-se ainda uma relação exposto/não exposto aos fatores de menor prevalência de 1:2, o risco de morte específico do grupo de recém- 
nascidos estudados de $10,0 \% 8$ e um risco relativo de morte 2,0 para os fatores estudados com base na literatura 9 . O número total de recém-nascidos assim calculado foi de 507.

\section{Coleta de dados}

Os seguintes dados foram coletados do prontuário médico:

(1) peso ao nascimento foi aferido na sala de parto ou na admissão do recém-nascido no berçário ou na UTI e estratificado com intervalos de 250g;

(2) idade gestacional - a avaliação da idade gestacional foi feita pela data da última menstruação materna, no caso da inexistência dessa informação no prontuário, a idade adotada foi a informada pelo obstetra ou pela avaliação do neonatologista, pelo método de New Ballard 10, no primeiro exame físico;

(3) tempo de morte: número de dias que ocorreu o óbito; neonatal precoce (até 6 dias completos) e tardia (entre 7 e 27 dias completos);

(4) a causa de óbito registrada no prontuário médico do recém-nascido seria aquela descrita como a causa conseqüêncial da morte no atestado de óbito.

A entrevista e a coleta dos dados do prontuário foram feitas por médicos do serviço de pediatria das instituições incluídas e supervisionadas pelo coordenador da pesquisa. Um médico de cada unidade estudada teve a tarefa de supervisionar o trabalho local. O pesquisador visitou semanalmente as maternidades, juntamente com o médico entrevistador, para supervisão e avaliação do trabalho desenvolvido.

Após o estudo-piloto, que foi realizado em agosto e setembro de 2001 nas quatro instituições que colaboram no projeto e que teve como objetivo avaliar os instrumentos de coleta e a organização do trabalho de campo, foi possível adequar os procedimentos previstos pelo estudo.

Foi incluído, assim, no estudo-piloto um total de 44 recém-nascidos. Ao final da fase-piloto, observou-se que o maior número de informações ignoradas foi relativo à história materna, especialmente no recém-nascido morto com menos de 24 h de vida, devido à alta rápida da mãe, o que dificultou a coleta de dados maternos. A partir desse período foram adotadas medidas para melhorar a coleta de dados e diminuir as perdas de informações fornecidas pelas mães, como, na Instituição 4, em que houve visitas do entrevistador em dias alternados durante a semana.

Os instrumentos se mostraram adequados. Os dados encontrados no estudo-piloto foram incluídos na análise final do estudo.

\section{Análise dos dados}

Inicialmente foi realizada a descrição dos dados levantados por maternidade que incluiu a distribuição dos recém-nascidos vivos, dos recém-nascidos de muito baixo peso, do percentual de óbitos em cada instituição. As causas de morte foram transcritas em ordem decrescente como mortalidade neonatal total e seus componentes. Em seguida, foram calculados os coeficientes de mortalidade neonatal total, precoce e tardio por instituição. Foram calculados os coeficientes de mortalidade estratificados por peso ao nascimento e idade gestacional. A estimação Kaplan-Meier pela análise de sobrevida foi realizada para se comparar a mortalidade entre os recém-nascidos de muito baixo peso de menor peso, entre 500 e 999g, com o de maior peso, entre 1.000 e $1.500 \mathrm{~g}$ e calculado o teste de log-rank.

Toda a análise estatística foi feita no programa Stata, versão 6.0.

\section{Resultados}

Foram incluídos no estudo um total de 509 recém-nascidos de muito baixo peso. Destes foram excluídos 17 recém-nascidos transferidos para outras instituições não participantes do estudo $(3,3 \%$ do total de recém-nascidos estudados) e cinco recém-nascidos com malformação. A amostra final constou, assim com 487 recém-nascidos $(96,0 \%)$ da amostra inicialmente planejada.

A Tabela 1 mostra o total de nascimentos por maternidade e o respectivo percentual de recém-nascidos de muito baixo peso e óbitos. A Instituição 1 apresentou o menor número de recém-nascidos vivos e o maior percentual de recém-nascidos de muito baixo peso. A Instituição 4 mostrou o maior número de recémnascidos vivos e o menor percentual de recémnascidos de muito baixo peso. A Instituição 3 mostrou o maior percentual de óbitos; a Instituição 2, o menor.

A Tabela 2 mostra os coeficientes de mortalidade neonatal e, em separado, os componentes precoce e tardio. Com exceção da Instituição 2, observa-se o predomínio da mortalidade neonatal precoce. As instituições 1 e 3 mostraram as taxas mais elevadas de mortalidade neonatal precoce. A Instituição 4 mostrou a menor taxa de mortalidade neonatal tardia.

A mesma tabela mostra uma grande variação da mortalidade neonatal precoce, de 13,5\%o na Instituição 1 a 2,0\%o na Instituição 2 . A Instituição 1 apresentou o maior coeficiente de 


\section{Tabela 1}

Total de nascimentos, número de recém-nascidos de muito baixo peso e percentagem de óbitos por instituição em quatro maternidades. Município do Rio de Janeiro, Brasil, no período entre 28 de agosto de 2001 a 28 de setembro de 2002 .

\begin{tabular}{lcrrrr}
\hline Instituição* & $\begin{array}{c}\text { Total de } \\
\text { recém-nascidos }\end{array}$ & \multicolumn{4}{c}{$\begin{array}{c}\text { Recém-nascidos de } \\
\text { muito baixo peso** } \\
\text { Total }\end{array}$} \\
& $n$ & $n$ & $\%$ & $n$ & $\%$ \\
\hline 1 & 667 & 42 & 6,70 & 11 & 26 \\
2 & 990 & 59 & 6,05 & 6 & 10 \\
3 & 4.216 & 196 & 4,64 & 72 & 37 \\
4 & 5.393 & 190 & 3,82 & 37 & 19 \\
Total & 11.266 & 487 & 4,32 & 126 & 26 \\
\hline
\end{tabular}

* Instituição 1: Hospital Universitário Pedro Ernesto da Universidade do Estado do Rio de Janeiro; Instituição 2: Instituto Fernandes Figueira do Ministério da Saúde; Instituição 3: Instituto Municipal da Mulher Fernando Magalhães; Instituição 4: Hospital Maternidade Alexandre Fleming;

** Peso inferior a $1.500 \mathrm{~g}$

Tabela 2

Coeficientes de mortalidade neonatal por instituição em quatro maternidades. Município do Rio de Janeiro, Brasil, no período entre 28 de agosto de 2001 a 28 de setembro de 2002 .

\begin{tabular}{lccccc}
\hline Mortalidade & \multicolumn{5}{c}{ Coeficientes de mortalidade* } \\
& 1 & 2 & 3 & 4 & Total \\
\hline Instituição** & 16,5 & 6,0 & 17,1 & 7,0 & 11,2 \\
Preconatal & 13,5 & 2,0 & 12,6 & 6,0 & 8,3 \\
1으 dia de vida & 9,0 & 1,0 & 6,0 & 2,0 & 3,6 \\
2ㅇ-6o dia de vida & 4,5 & 1,0 & 6,6 & 4,0 & 4,7 \\
Tardia & 3,0 & 4,0 & 4,5 & 1,0 & 2,9
\end{tabular}

* Por mil nascidos vivos;

** Instituição 1: Hospital Universitário Pedro Ernesto da Universidade do Estado do Rio de Janeiro; Instituição 2: Instituto Fernandes Figueira do Ministério da Saúde; Instituição 3: Instituto Municipal da Mulher Fernando Magalhães; Instituição 4: Hospital Maternidade Alexandre Fleming.
A Tabela 4 mostra a mortalidade por faixa de peso ao nascimento e idade gestacional. Os resultados mostraram o percentual inferior a $50,0 \%$ de mortalidade, nas faixas de peso, ao nascimento, superior a 500 e $749 \mathrm{~g}$ e a idade gestacional superior à faixa entre 24 e 26 semanas.

A Tabela 5 apresenta o tempo de morte, em dias, dos recém-nascidos de muito baixo peso. Para todas as faixas de peso, o percentual de morte é maior no primeiro dia de vida seguindo com uma diminuição até o sexto dia quando retorna a elevar. Observamos que independente da faixa de peso os percentuais de óbitos encontrados para cada dia foram semelhantes.

Quando os dados são analisados apenas para os recém-nascidos com peso inferior a $1.000 \mathrm{~g}$, observou-se que persistiu a mortalidade maior no primeiro dia de vida, diminuindo até o $6 \underline{0}$ dia e uma elevação entre o 7o e 28o dias de vida (Tabela 6).

Na estimação Kaplan-Meier pela análise de sobrevida (Figura 1), comparando as faixas de peso ao nascimento entre $500 \mathrm{e} 999 \mathrm{~g}$ às faixas entre 1.000 e $1.500 \mathrm{~g}$, observamos que $72,5 \%$ e $77,0 \%$, respectivamente, das mortes ocorrem até o 6 o dia completo de vida e no primeiro dia apenas $33,0 \%$ e $36,0 \%$, respectivamente. Do $7 \underline{\text { o até o }}$ 21 o dias de vida ainda se observa uma gradual queda da curva e uma estabilização do 22o ao 28 o dias de vida. A mortalidade menor de 10,0\% apenas é alcançada após 22o dia de vida. As curvas não foram estatisticamente significativas, $o$ teste de log-rank mostrou valor de $\mathrm{p}=0,7$.

A Tabela 7 apresenta a distribuição das causas de óbito para mortalidade neonatal precoce e tardia com o total de recém-nascidos estudados. Nos primeiros seis dias as causas de óbitos, em ordem de freqüência, foram a doença da membrana hialina, a sepsis, a hemorragia pulmonar, a asfixia perinatal, pneumotórax e a pneumonia congênita.

Entre o 7ㅇ e o 27ㅇ dias de vida, a causa de morte mais freqüente foi a sepsis, sendo seguida pela pneumonia congênita.

mortalidade neonatal precoce, mostrando uma grande proporção de óbitos no primeiro dia de vida $(62,5 \%)$. A menor taxa de mortalidade neonatal tardia foi observada na Instituição 4. A Instituição 3 foi aquela que mostrou os valores mais elevados de mortalidade neonatal tardia.

A Tabela 3 apresenta os percentuais de morte entre os recém-nascidos de muito baixo peso, a diferença para a Tabela 4 seria o predomínio da mortalidade neonatal precoce na Instituição $3(27,0 \%)$ quando comparada com a Instituição $1(21,4 \%)$.

\section{Discussão}

Os recém-nascidos de muito baixo peso representam um pequeno número no total de nascimentos, mas corresponderam à metade do número de mortes no período neonatal. Sendo assim, para que haja uma diminuição da mortalidade neonatal, sobretudo em sua fase precoce, é fundamental se investir na assistência desse grupo de recém-nascidos.

Utilizando os dados da Secretaria Municipal de Saúde do ano de 2002 11, a amostra estu- 
Percentuais de morte entre os recém-nascidos de muito baixo peso em quatro maternidades.

Município do Rio de Janeiro, Brasil, no período entre 28 de agosto de 2001 a 28 de setembro de 2002.

\begin{tabular}{|c|c|c|c|c|c|c|c|c|c|c|}
\hline \multirow[t]{3}{*}{ Mortalidade } & \multicolumn{10}{|c|}{ Instituição* } \\
\hline & \multicolumn{2}{|c|}{1} & \multicolumn{2}{|c|}{2} & \multicolumn{2}{|c|}{$3^{3}$} & \multicolumn{2}{|c|}{4} & \multicolumn{2}{|c|}{ Total } \\
\hline & $n$ & $\%$ & $\mathrm{n}$ & $\%$ & $n$ & $\%$ & $n$ & $\%$ & $n$ & $\%$ \\
\hline Neonatal & 11 & 26,2 & 6 & 10,2 & 73 & 36,7 & 37 & 19,4 & 126 & 25,9 \\
\hline Precoce & 9 & 21,4 & 2 & 3,4 & 53 & 27,0 & 31 & 16,2 & 94 & 19,3 \\
\hline Tardia & 2 & 4,8 & 4 & 6,8 & 19 & 9,7 & 6 & 3,2 & 32 & 6,6 \\
\hline
\end{tabular}

* Instituição 1: Hospital Universitário Pedro Ernesto da Universidade do Estado do Rio de Janeiro; Instituição 2: Instituto Fernandes Figueira do Ministério da Saúde; Instituição 3: Instituto Municipal da Mulher Fernando Magalhães; Instituição 4: Hospital Maternidade Alexandre Fleming.

dada nas quatro maternidades correspondeu a $18,0 \%$ do total dos recém-nascidos vivos, a $52,0 \%$ dos recém-nascidos de muito baixo peso vivos e a $34,0 \%$ das mortes em recém-nascidos de muito baixo peso atendidos na rede pública municipal. Nossos resultados mostraram um percentual de $4,3 \%$ de recém-nascidos de muito baixo peso entre o total de recém-nascidos vivos. No Município do Rio de Janeiro 3, a mesma incidência no ano de 2002 , foi de $1,6 \%$. O elevado percentual de recém-nascidos de muito baixo peso, entre as instituições estudadas, pode ser explicado por tratarem-se de maternidades com atendimento de nível terciário; por isso, receberam gestantes e recém-nascidos de alto risco.

A mortalidade neonatal em recém-nascidos de muito baixo peso foi de $11,2 \%$, sendo o componente precoce de $8,3 \%$ e tardio de $2,9 \%$. Os dados obtidos no Município do Rio de Janeiro 3 mostraram a mortalidade neonatal para todos recém-nascidos igual a $10,5 \%$, sendo o componente precoce de $8,0 \%$ e o tardio de $2,5 \%$. A mortalidade neonatal em recém-nascidos de muito baixo peso foi de $6,0 \%$. Os coeficientes de mortalidade encontrados, quando comparados aos de países desenvolvidos, mostraramse mais elevados, evidenciando a importância da mortalidade neonatal precoce no total da mortalidade neonatal.

O estudo de Gould 12 , realizado na Califórnia, em 1993, apresentou uma mortalidade neonatal de recém-nascidos de muito baixo peso igual a $1,6 \%$, sendo a mortalidade neonatal precoce de $1,3 \%$ e a tardia de $0,3 \%$. Nesse estudo a incidência de recém-nascidos de muito baixo peso foi de $0,9 \%$. Em estudo realizado na Finlândia 7, no ano de 2000, a mortalidade neonatal precoce foi de $2,0 \%$.

Em nosso estudo, a mortalidade específica encontrada em recém-nascidos de muito baixo

\begin{tabular}{|c|c|c|}
\hline \multicolumn{3}{|c|}{$\begin{array}{l}\text { Percentual de óbitos em cada faixa de peso } \\
\text { ao nascimento e a idade gestacional. }\end{array}$} \\
\hline & \multicolumn{2}{|c|}{ Óbitos } \\
\hline & $\mathrm{n}$ & $\%$ \\
\hline \multicolumn{3}{|c|}{ Peso nascimento $(\mathrm{g})$} \\
\hline 500-749 & 45 & 77,59 \\
\hline $750-999$ & 39 & 32,17 \\
\hline $1.000-1.249$ & 28 & 20,29 \\
\hline $1.250-1.500$ & 16 & 9,09 \\
\hline Total & 126 & \\
\hline \multicolumn{3}{|c|}{ Idade gestacional (semanas) } \\
\hline $24-26$ & 33 & 66,00 \\
\hline $27-28$ & 29 & 39,73 \\
\hline $29-30$ & 11 & 28,21 \\
\hline$>30$ & 52 & 16,05 \\
\hline Total & 125 & 25,72 \\
\hline
\end{tabular}

peso foi de $259,0 \%$, e ao compararmos tal resultado aos resultados de autores americanos, apresentados nos estudos de Gould 12 e Guyer 13 , respectivamente, $170,0 \%$ e $162,0 \%$, confirmamos que nossos resultados foram bastantes elevados. No entanto, os resultados de um estudo realizado na cidade de Montevidéu, Uruguai, em 1999, apresentaram um índice de mortalidade superior a $350,0 \%$ o 14 , bem acima ao apresentado em nossos estudos. Os nossos resultados também se mostraram inferiores aos publicados pelo Município do Rio de Janeiro que foi de $376,0 \%$, durante o ano de 20023. Apesar dos nossos resultados serem elevados, a mortalidade do município é ainda maior, mostrando que há outras unidades neonatais com uma assistência pior. 
Tabela 5

Tempo de morte dos recém-nascidos de muito baixo peso.

\begin{tabular}{|c|c|c|c|c|c|c|c|c|}
\hline \multirow[t]{3}{*}{ Óbito } & \multicolumn{8}{|c|}{ Peso ao nascimento $(\mathrm{g})$} \\
\hline & \multicolumn{2}{|c|}{$500-749$} & \multicolumn{2}{|c|}{ 751-999 } & \multicolumn{2}{|c|}{$1.000-1.500$} & \multicolumn{2}{|c|}{$500-1.500$} \\
\hline & $\%$ & Cumulativo & $\%$ & Cumulativo & $\%$ & Cumulativo & $\%$ & Cumulativo \\
\hline 1으 dia & 33,3 & 33,3 & 32,4 & 32,4 & 36,4 & 36,4 & 34,1 & 34,1 \\
\hline 2o dia & 20,0 & 53,3 & 18,9 & 51,3 & 4,5 & 40,9 & 14,3 & 48,4 \\
\hline 3o dia & 13,3 & 66,6 & 10,8 & 62,1 & 11,4 & 52,3 & 11,9 & 60,3 \\
\hline 4o dia & 0,0 & 66,6 & 5,4 & 67,5 & 9,1 & 61,4 & 4,8 & 65,1 \\
\hline 5o dia & 2,2 & 68,8 & 0,0 & 67,5 & 2,3 & 63,7 & 1,6 & 66,7 \\
\hline 6o dia & 2,2 & 71,0 & 8,1 & 75,6 & 13,6 & 77,3 & 7,9 & 74,6 \\
\hline 7ㅇ-27으 dia & 29,0 & 100,0 & 24,3 & 100,0 & 22,7 & 100,0 & 25,4 & 100,0 \\
\hline
\end{tabular}

Tabela 6

Tempo de morte em recém-nascidos com peso inferior a $1.000 \mathrm{~g}$.

\begin{tabular}{|c|c|c|c|c|c|c|}
\hline \multirow[t]{3}{*}{ Óbito } & \multicolumn{6}{|c|}{ Peso ao nascimento (g) } \\
\hline & \multicolumn{2}{|c|}{$500-749$} & \multicolumn{2}{|c|}{$751-999$} & \multicolumn{2}{|c|}{$500-999$} \\
\hline & $\%$ & Cumulativo & $\%$ & Cumulativo & $\%$ & Cumulativo \\
\hline 1으 dia & 33,3 & 33,3 & 32,4 & 32,4 & 33,0 & 33,0 \\
\hline 2o o dia & 20,0 & 53,3 & 18,9 & 51,3 & 19,5 & 52,5 \\
\hline 3으 dia & 13,3 & 66,6 & 10,8 & 62,1 & 12,0 & 64,5 \\
\hline 4o dia & 0,0 & 66,6 & 5,4 & 67,5 & 2,7 & 67,2 \\
\hline 5으 dia & 2,2 & 68,8 & 0,0 & 67,5 & 1,1 & 68,3 \\
\hline 6o dia & 2,2 & 71,0 & 8,1 & 75,6 & 5,2 & 72,5 \\
\hline 7ㅇ-27으 dia & 29,0 & 100,0 & 24,3 & 100,0 & 26,5 & 100,0 \\
\hline
\end{tabular}

Figura 1

Análise de sobrevivência pela estimação de Kaplan-Meier.

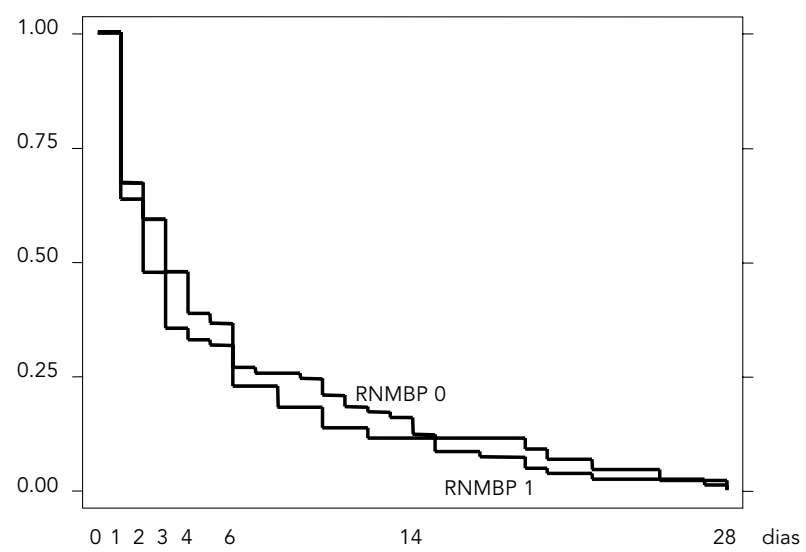

RNMBP 0 = Recém-nascidos de muito baixo peso $(500-999 \mathrm{~g})$

RNMBP 1 = Recém-nascidos de muito baixo peso (1.000-1.500g).
Os resultados encontrados por Leal \& Szwarcwald 1, que referiram uma alta mortalidade neonatal precoce, sendo uma das prováveis causas de morte a diminuição do número de leitos hospitalares disponíveis na rede pública, retardando-se assim a hospitalização da parturiente, o que levaria a um sofrimento fetal.

$\mathrm{O}$ estudo da mortalidade neonatal, no $\mathrm{Mu}$ nicípio do Rio de Janeiro, no período entre 1994 e 2000, apresentou uma redução, que Gomes 4 menciona que foi provocada por uma melhora dos motivos que referiram Leal \& Szwarcwald 1 anteriormente. O Município do Rio de Janeiro incorporou maternidades estaduais e federais para sua gerência aumentando o número de leitos hospitalares para gestantes e recém-nascidos de alto risco, chegando ao percentil de $35,0 \%$ de todos os nascimentos no município. O município promoveu: (a) aumento de recursos materiais como o uso de novas tecnologias para tratamento, tanto das gestantes quanto dos recém-nascidos de risco; e (b) aumento e treinamento de recursos humanos.

A faixa de idade gestacional em que se iniciou a melhor relação de sobrevivência/mortalidade (viabilidade) foi após intervalo entre 24 e 26 semanas de gestação, valor superior em duas semanas gestacionais aos encontrados em países desenvolvidos 5,15,16. Observamos que esse patamar, a partir de 27 semanas de gestação, poderia estar supervalorizado porque houve um predomínio da avaliação da idade gestacional pelo método de New Ballard 10, o qual utiliza o exame físico do recém-nascido pelo neonatologista. Esse método é questionado por vários autores 5,17,18, que também observaram uma superestimação da idade gestacional até quatro semanas de gestação.

Já a faixa de peso ao nascimento na qual se iniciou a viabilidade foi superior à faixa entre 
Distribuição das causas de óbito dos recém-nascidos de muito baixo peso.

\begin{tabular}{|c|c|c|c|c|c|c|}
\hline \multirow[t]{3}{*}{ Causa de morte } & \multicolumn{6}{|c|}{ Mortalidade neonatal } \\
\hline & \multicolumn{2}{|c|}{ Precoce } & \multicolumn{2}{|c|}{ Tardia } & \multicolumn{2}{|c|}{ Total } \\
\hline & $n$ & $\%$ & $\mathrm{n}$ & $\%$ & $\mathrm{n}$ & $\%$ \\
\hline Sepsis & 17 & 40,0 & 26 & 60,0 & 43 & 34,0 \\
\hline Doença membrana hialina & 34 & 100,0 & 0 & 0,0 & 34 & 27,0 \\
\hline Hemorragia pulmonar & 14 & 100,0 & 0 & 0,0 & 14 & 11,0 \\
\hline Asfixia perinatal & 12 & 92,0 & 1 & 8,0 & 13 & 10,3 \\
\hline Pneumonia congênita & 4 & 57,0 & 3 & 43,0 & 7 & 5,5 \\
\hline Pneumotórax & 5 & 100,0 & 0 & 0,0 & 5 & 4,0 \\
\hline Insuficiência renal & 2 & 50,0 & 2 & 50,0 & 4 & 3,2 \\
\hline Imaturidade & 2 & 100,0 & 0 & 0,0 & 2 & 1,6 \\
\hline Hemorragia intracraniana & 2 & 100,0 & 0 & 0,0 & 2 & 1,6 \\
\hline Enterocolite necrosante & 1 & 100,0 & 0 & 0,0 & 1 & 0,8 \\
\hline Coagulação intravascular disseminada & 1 & 100,0 & 0 & 0,0 & 1 & 0,8 \\
\hline Total & 94 & 73,0 & 32 & 27,0 & 126 & 100,0 \\
\hline
\end{tabular}

500 e 749g, valor bem próximo aos de países desenvolvidos 15,16. A mensuração do peso foi muito mais fidedigna do que a avaliação da idade gestacional. Os resultados indicam que não seria a imaturidade a causa principal das altas taxas de mortalidade, visto que mostramos uma viabilidade de peso, ao nascimento, e idade gestacional bem próxima à dos países desenvolvidos.

Foi observado que até $72 \mathrm{~h}$ de vida, a taxa de óbito foi menor do que a observada por outros estudos 6,19,20, especialmente devido a uma baixa mortalidade no primeiro dia de vida. $\mathrm{O}$ mesmo resultado foi encontrado por Gomes 4 em estudo realizado, também, em maternidades no Município do Rio de Janeiro.

A análise de sobrevida mostrou uma mortalidade baixa no primeiro dia de vida tanto na faixa de peso entre 500 e $999 \mathrm{~g}$ quanto entre 1.000 e $1.500 \mathrm{~g}$. A taxa de mortalidade acumulativa em nosso estudo para os recém-nascidos com peso inferior a $1.000 \mathrm{~g}$ foi no primeiro dia de $33,0 \%$, segundo dia $52,5 \%$, terceiro dia $64,5 \%$ e quarto dia $65,1 \%$. O estudo de Meadow 6 mostrou os valores $45,0 \%, 61,0 \%, 76,0 \%$ e $85,0 \%$, respectivamente. Valores maiores do que os encontrados em nosso estudo. Nossos resultados mostraram que os recém-nascidos de muito baixo peso estariam morrendo num período mais tardio, sobretudo se compararmos o período após o quarto dia de vida, quando no estudo de Meadow 6 a mortalidade é de $19,0 \%$, no nosso estudo revelou-se uma mortalidade elevada, de $38,9 \%$ em recém-nascidos com peso ao nascimento inferior a $1.000 \mathrm{~g}$.
Nossos resultados demonstraram como principal causa de mortalidade neonatal a sepsis (34,0\%); se considerarmos a pneumonia congênita também como sepsis a freqüência de óbitos com essa classificação aumentaria para $40,0 \%$. Em segundo lugar se apresentou a doença da membrana hialina $(28,0 \%)$, em terceiro a hemorragia pulmonar $(11,0 \%)$ e em quarto a asfixia perinatal $(10,0 \%)$. As mortes com diagnóstico de sepsis predominaram durante o período da mortalidade neonatal tardia $(58,0 \%)$, mas não deixaram de ser importantes na mortalidade neonatal precoce, aí ocupando o segundo lugar. Os resultados encontrados mostraram uma semelhança com o estudo de Barton 19, que relataram a importância das infecções congênitas como a principal causa da mortalidade neonatal. O diagnóstico de sepsis foi diferente nos dois estudos: no nosso o diagnóstico foi clínico, e no de Barton 19 foi por necropsia. Outros autores 21,22,23 já haviam relatado a importância das infecções congênitas como a principal causa de morte, mormente nos primeiros sete dias de vida.

Philip 5 e Lemons et al. 8 mostraram uma sobrevida de $90,0 \%$ para recém-nascidos com peso, ao nascimento, superior a $750 \mathrm{~g}$. No presente estudo, o mesmo nível de sobrevida foi encontrado na faixa de peso, ao nascimento, superior a $1.249 \mathrm{~g}$. Verificamos que, se retirarmos a faixa de peso em que a sobrevida ainda é menor do que $50,0 \%$, especialmente devido à imaturidade (morte inevitável), a maior mortalidade se daria na faixa de peso entre $750 \mathrm{e}$ $1.249 \mathrm{~g}$, justamente a mesma faixa de peso em que ocorreu o pico da sepsis. 
Nosso estudo concluiu que a menor mortalidade nos primeiros dias de vida teria se dado em virtude da melhoria na assistência ao parto, do aumento do número de leitos, da elevação de recursos materiais com tecnologias modernas, da padronização de conduta no atendimento, da obrigatoriedade da presença do pediatra na sala de parto e do aumento e treinamento dos profissionais envolvidos na assistência. Mas com o prosseguimento da assistência ao recém-nascido, esse estaria se contaminando e assim, transferindo o predomínio da mortalidade dos primeiros dias para mais tardiamente.

A maior limitação do nosso estudo foi a impossibilidade do desfecho, causa de óbito, não ter sido diagnosticado por estudo anatomopatológico. A principal causa de óbito relatada foi a infecção neonatal, cuja principal etiologia seria a contaminação hospitalar.

Com exceção da Instituição 2, todas as outras instituições mostraram um predomínio da mortalidade neonatal precoce. A Instituição 1 foi quem apresentou a maior quantidade de morte no primeiro dia de vida mostrando uma provável deficiência na atenção no atendimento pré-natal e ao parto. A Instituição 3 apresentou o pior desempenho, tanto na mortalidade neonatal precoce quanto tardia, demonstrando uma deficiência na assistência ao pré-natal, ao parto e ao recém-nascido. A Instituição 4 apresentou o mais baixo coeficiente de mortalidade neonatal tardia, podendo ser devido a transferências dos recém-nascidos para outros hospitais a fim de evitar a superpopulação. A Instituição 2 foi quem obteve menor coeficiente de mortalidade neonatal e o predomínio do componente tardio por apresentar algumas ca- racterísticas especiais: primeiro pela localização em bairro de classe social alta, o que dificulta o acesso da população carente e, segundo, por apresentar o melhor desempenho quantos aos fatores de proteção como o maior percentil de visitas ao pré-natal, o uso de corticosteróide antenatal e o uso de surfactante pulmonar 24 .

\section{Conclusões}

Os coeficientes de mortalidade neonatal em recém-nascidos de muito baixo peso foram bastante elevados. A viabilidade encontrada foi superior às faixas de peso entre 500 e $749 \mathrm{~g}$ e à faixa de idade gestacional entre 24 e 26 semanas. O tempo de morte foi menor nos primeiros dias de vida devido a uma maior mortalidade mais tardia. Predominou como causa de morte a infecção neonatal. As ações de uma política para diminuição da mortalidade neonatal deveriam priorizar o controle das infecções perinatais, em cada instituição, por intermédio de uma comissão de controle de infecção hospitalar atuante e respeitada. O estudo anatomopatológico rotineiro de todas as placentas e de necropsia de recém-nascidos de muito baixo peso deveria ser obrigatório. Investimento em recursos materiais e humanos para o aumento do número de leitos para evitar superpopulação e, com isso, a infecção hospitalar por microorganismos multirresistentes. Presença obrigatória e permanente de neonatologista tanto no atendimento ao parto quanto na unidade de tratamento intensivo e na unidade intermediária.

\section{Resumo}

O objetivo foi descrever as causas de morte em recémnascidos de muito baixo peso, em quatro maternidades do Município do Rio de Janeiro, Brasil. No estudo longitudinal foram incluídos todos os recém-nascidos de muito baixo peso até completarem 27 dias de vida. Descrita a distribuição dos recém-nascidos vivos, recém-nascidos de muito baixo peso, percentual de óbitos por maternidades e o seu total, foram calculados os coeficientes de mortalidade neonatal total e seus componentes. Viabilidade e tempo de mortes foram avaliados. O estudo incluiu 487 recém-nascidos. A mortalidade neonatal foi de 11,3\%o, a precoce de 8,3\% e a tardia de 2,9\%o. A viabilidade de recém-nascidos de muito baixo peso foi superior a 26 semanas gestacionais e $749 \mathrm{~g}$ de peso. A mortalidade foi baixa nos primeiros três dias de vida, especialmente no 1o, e elevada do 4 o ao 28o. Principal causa da morte foi a sepsis. As ações dos gestores de saúde devem ser direcionadas na melhoraria do diagnóstico; controle das infecções perinatais; diminuição da contaminação hospitalar dos recém-nascidos, com redução da superpopulação nas enfermarias; estudo rotineiro das placentas e necropsia de recém-nascidos de muito baixo peso.

Mortalidade Infantil; Recém-nascido de Muito Baixo Peso; Causa da Morte 


\section{Colaboradores}

J. L. M. B. Duarte e G. A. S. Mendonça participaram da elaboração do projeto, análise dos resultados e redação final do artigo. J. L. M. B. Duarte contribuiu na revisão da literatura.

\section{Referências}

1. Leal MC, Szwarcwald CL. Evolução da mortalidade neonatal no Estado do Rio de Janeiro, Brasil, de 1979 a 1993. 1 - Análise por grupo etário segundo região de residência. Rev Saúde Pública 1996; 30:403-12.

2. Menezes AMB, Barros FC, Victora CG, Alves C, Rocha C, Albernaz E, et al. Mortalidade perinatal em duas coortes de base populacional no Sul do Brasil: tendências e diferenciais. Cad Saúde Pública 1996; 12 Suppl 1:33-41.

3. Gerência de Informações Epidemiológicas, Coordenação de Epidemiologia, Superintendência de Saúde Coletiva, Secretaria Municipal do Rio de Janeiro. Taxa de mortalidade infantil, perinatal e fetal no Município do Rio de Janeiro - 1979 a 2002. Rio de Janeiro: Secretaria Municipal de Saúde do Rio de Janeiro; 2004.

4. Gomes MA. Assistência Neonatal na Secretaria Municipal de Saúde do Rio de Janeiro: uma análise do período 1995-2000 [Tese de Doutorado]. Rio de Janeiro: Instituto Fernandes Figueira, Fundação Oswaldo Cruz; 2002.

5. Philip AG. Neonatal mortality rate: is further improvement possible? J Pediatr 1995; 126:427-33.

6. Meadow W, Reimshisel T, Lantos J. Birth weightspecific mortality for extremely low birth weight infants vanishes by four days of life: epidemiology and ethics in the neonatal intensive care unit. Pediatrics 1996; 97:636-43.

7. Tommiska V, Heinonen K, Ikonen S, Kero P, Pokela ML, Renlund M, et al. A national short-term followup study of extremely low birth weight infants born in Finland in 1996-1997. Pediatrics 2001; 107:E2.

8. Lemons JA, Bauer CR, Oh W, Korones SB, Papile LA, Stoll BJ, et al. Very low birth weight outcomes of the National Institute of Child health and human development neonatal research network, January 1995 through December 1996. NICHD Neonatal Research Network. Pediatrics 2001; 107:E1.

9. Grupo Colaborativo de Estudos Perinatais. Fatores perinatais relacionados com a morbidade e a mortalidade de recém-nascidos pertencentes a nove unidades neonatais do Município de São Paulo. J Pediatr (Rio J) 1996; 72:379-87.

10. Ballard JL, Novak KK, Driver M. A simplified score for assessment of fetal maturation of newly born infants. J Pediatr (Rio J) 1979; 95 (5 Pt 1):769-74.

11. Gerência de Informações Epidemiológicas, Coordenação de Epidemiologia, Superintendência de Saúde Coletiva, Secretaria Municipal do Rio de Janeiro. Taxa de mortalidade neonatal peso-específica no Município do Rio de Janeiro - 1996 a 2002. Rio de Janeiro: Secretaria Municipal de Saúde do Rio de Janeiro; 2004

12. Gould JB, Benitz WE, Liu H. Mortality and time to

\section{Agradecimentos}

À Dra. Maria Rita Rocha Quadra e à Dra. Letícia Duarte Villela agradecemos a colaboração na coleta de dados.

death in very low birth weight infants: California 1987 and 1993. Pediatrics 2000; 105:E37.

13. Guyer B, Freedman MA, Strobino DM, Sondik EJ. Annual summary of vital statistics: trends in the health of Americans during the 20th century. Pediatrics 2000; 106:1307-17.

14. Matijasevich A, Barros FC, Fortaleza CA, DíazRossello JL. Atenção à saúde de crianças de peso muito baixo ao nascer, em Montevidéu, Uruguai: comparação entre os setores público e privado. J Pediatr (Rio J) 2001; 77:313-20.

15. Horbar JD. The Vermont-Oxford Neonatal Network: integrating research and clinical practice to improve the quality of medical care. Semin Perinatol 1995; 19:124-31.

16. O'Shea TM, Klinepeter KL, Goldstein DJ, Jackson BW, Dillard RG. Survival and developmental disability in infants with birth weights of 501 to 800 grams, born between 1979 and 1994. Pediatrics 1997; 100:982-6.

17. Hack M, Horbar JD, Malloy MH, Tyson JE, Wright E, Wright L. Very low birth weight outcomes of the National Institute of Child Health and Human Development Neonatal Network. Pediatrics 1991; 87:587-97.

18. Moraes CL, Reichenheim ME. Validade do exame clínico do recém-nascido para a estimação da idade gestacional: uma comparação do escore New Ballard com a data da última menstruação e ultra-sonografia. Cad Saúde Pública 2000; 16:83-94.

19. Barton L, Hodgman JE, Pavlova Z. Causes of death in the extremely low birth weight infant. Pediatrics 1999; 103:446-51.

20. Araujo BF, Bozzetti MC, Tanaka ACA. Mortalidade neonatal precoce no Município de Caxias do Sul: um estudo de coorte. J Pediatr (Rio J) 2000; 76:200-6.

21. Nakamura Y, Hosokawa Y, Yano H, Nakashima N, Nakashima T, Komatsu Y, et al. Primary causes of perinatal death. An autopsy study of 1000 cases in Japanese infants. Hum Pathol 1982; 13:54-61.

22. Quinn PA, Butany J, Chipman M, Taylor J, Hannah W. A prospective study of microbial infection in stillbirths and early neonatal death. Am J Obstet Gynecol 1985; 151:238-49.

23. Naeye RL. Causes of perinatal mortality in the U.S. Collaborative Perinatal Project. JAMA 1977; 238:228-9.

24. Duarte JLMB, Mendonça GAS. Fatores associados à morte neonatal em recém-nascidos de muito baixo peso em quatro maternidades no Município do Rio de Janeiro, Brasil. Cad Saúde Pública 2005; 21:181-91.

Recebido em 19/Mai/2004

Versão final reapresentada em 14/Set/2004

Aprovado em 16/Set/2004 